\section{Elemental analysis of histologi- cal specimens: a method to unmask nano asbestos fibers}

\author{
M. Scimeca, ${ }^{1,2 *}$ A. Pietroiusti, ${ }^{1 *}$ F. Milano, ${ }^{3}$ \\ L. Anemona, ${ }^{1}$ A. Orlandi, ${ }^{1}$ L.T. Marsella, ${ }^{1}$ \\ E. Bonanno ${ }^{1,2}$ \\ 'Department of Biomedicine and \\ Prevention, University of Rome Tor \\ Vergata \\ ${ }^{2}$ TMALab s.r.l., Spin-off of University of \\ Rome Tor Vergata \\ ${ }^{3}$ Department of Experimental Medicine \\ and Surgery, University of Rome Tor \\ Vergata, Italy
}

\section{Abstract}

There is an increasing amount of evidence that nanoparticles may enhance toxicological potential in comparison to the same material in the bulk form. The aim of this study was to develop a new method to unmask asbestos nanofibers from Formalin-Fixed ParaffinEmbedded (FFPE) tissue. For the first time, in this study we applied Energy Dispersive X-ray (EDX) microanalysis through transmission electron microscopy to demonstrate the presence of asbestos nanofibers in histological specimens of patients with possible occupational exposure to asbestos. The diagnostic protocol was applied to 10 randomly selected lung cancer patients with no history of previous asbestos exposure. We detected asbestos nanofibers in close contact with lung cancer cells in two lung cancer patients with previous possible occupational exposure to asbestos. We were also able to identify the specific asbestos iso-type, which in one of the cases was the same rare variety used in the workplace of the affected patient. By contrast, asbestos nanofibers were not detected in lung cancer patients with no history of occupational asbestos exposure.

The proposed technique can represent a potential useful tool for linking the disease to previous workplace exposure in uncertain cases. Furthermore, Formalin-Fixed ParaffinEmbedded (FFPE) tissues stored in the pathology departments might be re-evaluated for possible etiological attribution to asbestos in the case of plausible exposure. Since diseases acquired through occupational exposure to asbestos are generally covered by workers' insurance in most countries, the application of the protocol used in this study may have also relevant social and economic implications.

\section{Introduction}

In recent years, a lot of research has been performed on the possible health effects of engineered nanomaterials (ENM). ${ }^{1}$ Among ENM, the most potentially dangerous are carbon nanotubes (CNTs), due to their fiber-like shape shared with asbestos fibers, they may cause asbestos-like diseases. ${ }^{2}$ Indeed, in some comparative experimental animal studies, CNTs showed injuring effects similar, or even higher than that of asbestos. ${ }^{3}$

One of the paradigms of nanotoxicology is that the material in the nanometric range becomes more toxic than the same material in the bulk form. ${ }^{4}$ In this light, although recent epidemiological data suggest an association of occupational exposure to asbestos fibers having a diameter $<0.25 \mu \mathrm{m}$ (i.e., asbestos fibers within or close to the nanometric range of 1$100 \mathrm{~nm}$ ) with lung cancer, ${ }^{5}$ no histological demonstration of this association is currently available. It may depend on the fact that diagnostic techniques used for the demonstration of asbestos fibers in the lung are based on light microscopy, and therefore fibers having a diameter in the nanometric range are out of the resolving power of the technique.

In this work, involving two lung cancer patients with possible occupational asbestos exposure, we show that the application of Energy Dispersive X-ray (EDX) microanalysis may allow the unequivocal demonstration of asbestos nanofibers tightly associated with lung cancer cells.

\section{Materials and Methods}

\section{Patients}

In this retrospective study, we re-evaluated 10 lung biopsies of lung cancer patients with a history of possible exposure to asbestos and 10 randomly selected lung cancer patients with no history of previous asbestos exposure. All experiments were approved by the ethical committee of the University of Rome Tor Vergata; in particular, each sample was anonymized and all unnecessary sensitive data of patients were deleted from clinical report.

\section{Histological analysis}

All biopsies were formalin-fixed and paraffin embedded; four $\mu \mathrm{m}$-thick sections were routinely stained with haematoxylin and eosin (H\&E) and the morphological study was performed by an expert pathologist ${ }^{6}$ (Figure $1 \mathrm{~A}-\mathrm{B}$ ).

\section{Immunohistochemistry}

The phenotype of lung cancer was characterized by the presence of the thyroid tran-
Correspondence: Prof. Elena Bonanno, Department of Biomedicine and Prevention, University of Rome Tor Vergata, Via Montpellier 1, 00133 Rome, Italy. Tel. +39.06.20903913.

E-mail: elena.bonanno@uniroma2.it

Keywords: Asbestos fibers; nanofibers; EDX microanalysis; Transmission Electron Microscopy; lung cancer; occupational exposure.

Contributions: MS, AP, EB, study conception and design, manuscript drafting; LA, case selection and morphological classification; FM, A0, LTM, participation in study and cases selection. All authors were involved in writing the paper and approved the submitted and published versions.

*These authors equally contributed to the work.

Conflict of interest: the authors declare no conflict of interest.

Funding: this work has been supported by the Grant from the Italian Ministry of Health (RF2009-1536665), the EU-FP7 MARINA project (grant agreement 263215) and the EU-FP7 NANoREG project (grant agreement 310584). This work has also been supported in part by FILAS Grant FILAS-SO-2011-1076.

Received for publication: 2 October 2015. Accepted for publication: 22 November 2015.

This work is licensed under a Creative Commons Attribution-NonCommercial 4.0 International License (CC BY-NC 4.0)

(C) Copyright M. Scimeca et al., 2016

Licensee PAGEPress, Italy

European Journal of Histochemistry 2016; 60:2573 doi:10.4081/ejh.2016.2573

scription factor 1 (TTF-1) and cytokeratin 7 (typically expressed by adeno-carcinomas) (Figure 1 C-D). Briefly, 3 - $\mu$ m-thick sections were pre-treated with EDTA citrate $\mathrm{pH} 7.8$ for $30 \mathrm{~min}$ at $95^{\circ} \mathrm{C}$ and then incubated respectively with rabbit monoclonal anti-Cytokeratin 7 for $30 \mathrm{~min}$ (1:100 clone OV-TL12/30; Novus Biologicals, Littleton, CO, USA) and rabbit monoclonal anti-TTF-1 for 30 min (1:100 clone SP141; Spring Bioscience, Pleasanton, CA, USA). Washing was performed with PBS $4 \%+$ Tween20 pH 7.6 (UCS diagnostic, Rome, Italy) reactions were revealed by a horseradish peroxidase - diaminobenzidine detection kit (UCS diagnostic). ${ }^{7}$

\section{Transmission Electron Microscopy}

FFPE tissues retrieval for ultrastructural and elemental analysis: flat slice embedding

H\&E sections were used in order to identify areas suspected to harbor pollutant fibers (Figure 2A). Selected areas were often charac- 
terized by small black deposits similar to carbon. Six mm serial sections were collected on histology super-frost plus slides (Figure 2B). These sections were de-paraffinized, $3 \times 15$ min. in xylene and hydrated by a series of incubations in 100\%, 95\%, 70\%, 30\% ethanol and phosphate buffer $0.1 \mathrm{M}$. Then, sections were washed with phosphate buffer $0.1 \mathrm{M}$, and dehydrated by a series of incubations in $30 \%, 50 \%$, $70 \%$, ethanol. Dehydration was continued by incubations in 95\% ethanol, absolute ethanol and propylene oxide. Tissues were incubated with 1:1 epon-propylene oxide solution for 30 min, 3:1 epon-propylene oxide solution for 30 $\mathrm{min}$, and epon absolute for $3 \mathrm{~h}$ (Figure 2C). Embedding beam capsules were placed over areas previously identified on H\&E section (Figure 2D) and incubated for $24 \mathrm{~h}$ at $60^{\circ} \mathrm{C}$. Finally, beam capsules were detached from the slide with thermal shock (liquid nitrogen) (Figure 2E). All samples were studied by TEM Hitachi H-7100. Counting was performed at a magnification between 15,000 and 20,000, which permits detection of fibrils as small as 1 $\mathrm{nm}$ in diameter.

\section{EDX microanalysis}

The EDX microanalysis is a technology that performs the elemental and chemical analysis of a sample in a transmission electron microscope. When the electron beam in an electron microscope hits a thin sample, some atoms of the sample will be excited or ionized. When they return into their ground state, they will emit characteristic $\mathrm{x}$-rays. The $\mathrm{x}$-ray emission at different wavelengths may then be measured by a photon-energy-sensitive detector.

The EDX detector system performs a simultaneous display of all mid-energy (1-20 keV) $\mathrm{x}$ rays collected during any individual analysis period. Therefore it is possible to detect those elements with N.A.>10. The minimal detectable elemental concentration, which requires some signal averaging, is approximately $0.1 \mathrm{mmol}$ per $\mathrm{kg}$ of dry specimen (i.e., $10 \mathrm{ppm})$, whereas spatial resolution ranges from about $10 \mathrm{~nm}$ to a few micrometers.

For the EDX microanalysis $100 \mathrm{~nm}$-thick unstained ultrathin sections were placed on specific copper grids. The EDX spectra were acquired by a Hitachi 7100FA transmission electron microscope and an EDX detector (Thermo Scientific, Waltham, MA USA) at an acceleration voltage of $75 \mathrm{KeV}$ and 12000 magnification. Spectra were semi-quantitatively analyzed by the Noram System Six software (Thermo Scientific) using the standardless Cliff-Lorimer k-factor method. ${ }^{8}$ The EDX microanalysis system was calibrated using the x-ray microanalysis standard (Micro-Analysis Consultants Ltd., Cambridgeshire UK). ${ }^{8}$ The sample standards used as controls were: Dural (Al, $\mathrm{Cu}, \mathrm{Mg}$ ), Apatite (Ca5(P04)3(OH)), Lead
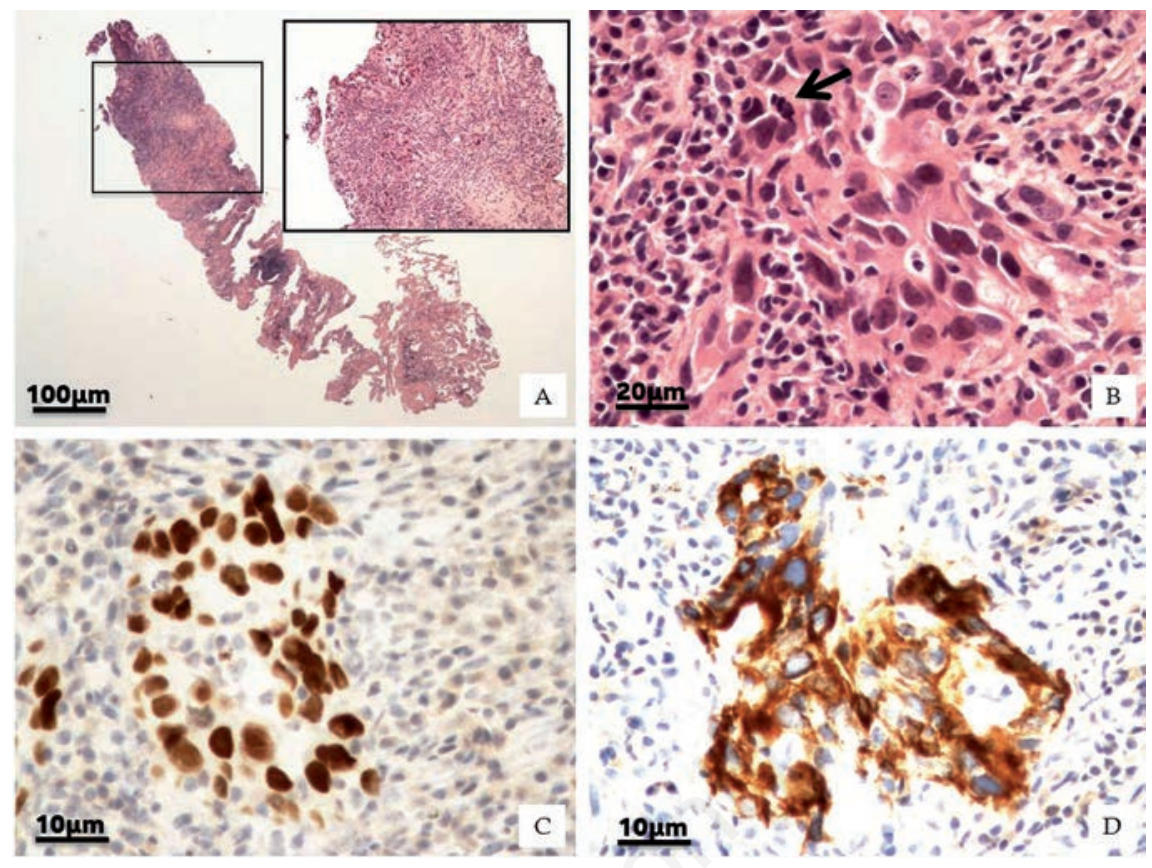

Figure 1. Lung histological classifications. A) H\&E of lung biopsy (2x). Square shows main lung lesion (10x). B) High magnification displays cohesive malignant cells with abundant cytoplasm, large nuclei and atypical mitosis (arrow) (40x). Neoplastic cells were characterized by nuclear expression of TTF-1 antigen (C) and CK7 positivity (D) $(40 x)$.

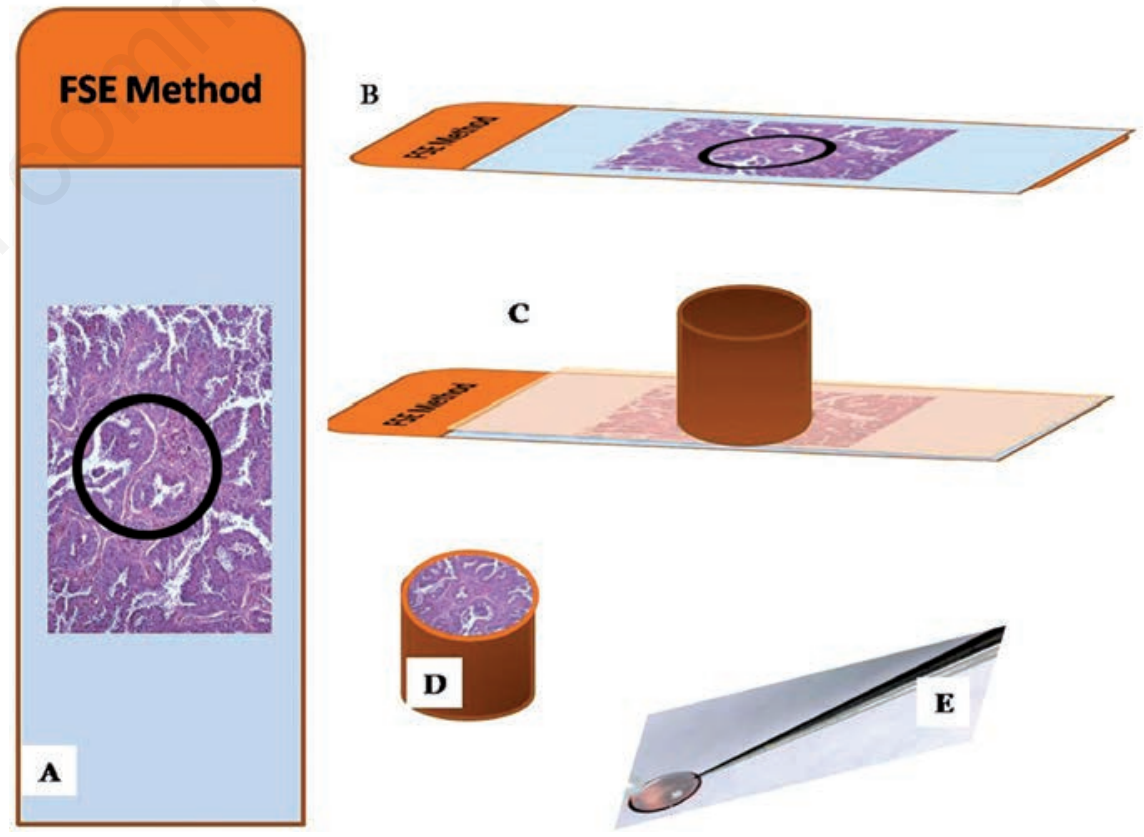

Figure 2. FSE method. A) H\&E stained sections were used to identify areas suspected to harbor pollutant fibers (circle). B) $6 \mu \mathrm{m}$-thick serial sections were collected on histology super-frost plus slides and processed for epon embedding. C) Embedding beam capsules were placed over areas previously identified. D) After incubation for $24 \mathrm{~h}$ at $60^{\circ} \mathrm{C}$, beam capsules were detached from the slide with thermal shock. E) Epon embedded tissue were cut and routinely stained with heavy metals solutions as uranyl acetate and lead citrate. 
Sulphide (PbS), Chromite (FeCr204), Chromium (Cr), Iron (Fe), Manganese (Mn), Silicon (Si), Tungsten (W), Zinc (Zn), Cadmium sulfide (CdS) and Cobalt (Co). This semi-quantitative approach pointed out the presence of asbestos fibers in the tissues.

\section{Results}

All lung biopsies were classified as lung adeno-carcinomas, according to the criteria released in 2012 from the World Health Organization (WHO). ${ }^{9}$ The observation of tissues by optical microscopy did not allow the identification of fibers related to contamination of asbestos. Then, we proceeded to TEM and EDX microanalysis evaluation in order to verify the presence of nano-fibers. The samples processed by the Flat slice embedding (FSE) method (Figure $3 \mathrm{C}$-D) presented a reduction of structural details, for lower magnifications $(<10,000 \mathrm{x})$ it was still possible to carry out morphological analysis. Actually, a strong morphological correlation was found between the selected area on H\&E slice and the corresponding area on the FSE embedded. In some of the FSE section, we detected nanofibers unnoticeable by optical microscopy. Noteworthy, corrosive substances used during tissues processing, such as paraformaldehyde, and propylene oxide do not damage sections and/or extract particles or fibers from them.

In fact, in adeno-carcinoma tissues of the two patients with a history of possible exposure to asbestos, we observed several fibers with a length variable from 0.1 to $0.6 \mu \mathrm{m}$ and a diameter below $0.1 \mu \mathrm{m}$ (Figure 4). In adenocarcinoma tissues of 10 randomly selected patients with no history of previous asbestos exposure, no fibers or nanofibers were detected at TEM analysis. In the two lung cancer patients with possible occupational exposure to asbestos, The EDX microanalysis showed that the identified nanofibers were mainly composed of silicon and iron (Figure 4 E-F). The association of morphometric and microanalytical data pointed out to the identification of the nanofibers as being composed of asbestos. In particular, fibers detected into the two lung adeno-carcinomas were compatible with an asbestiform variety of amosite, the gedrite (Figure 4).

\section{Discussions}

The two most relevant asbestos related disorders are pleural mesothelioma and lung cancer. The association between asbestos and lung cancer is still debated, ${ }^{10}$ given the typical-

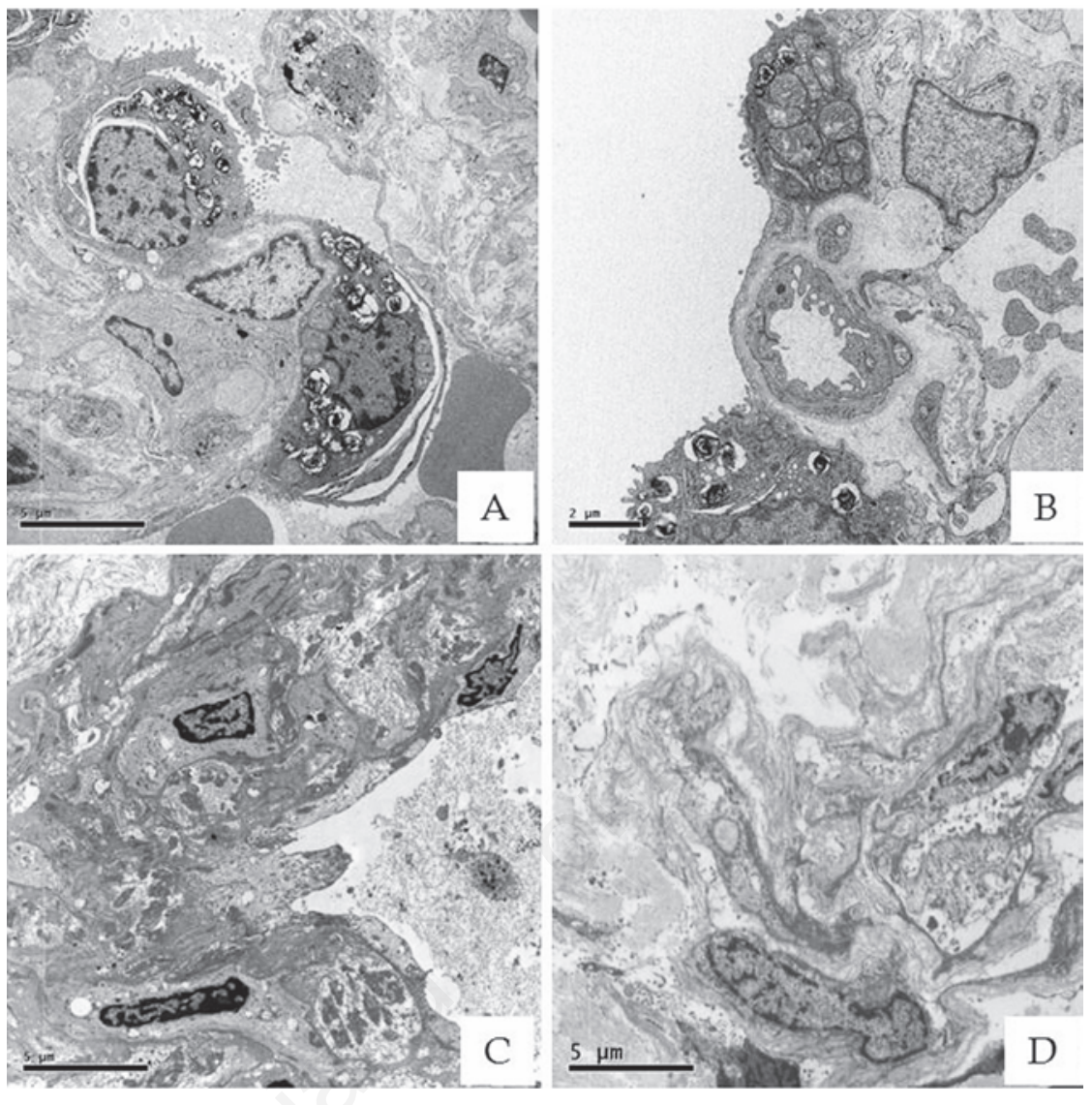

Figure 3. Ultrastructural preservation of FSE Epon embedded tissues. A-B) Lung tissues processed by standard transmission electron microscope protocol $(5000 x)$. C-D) Lung samples processed by FSE method showed a satisfactory preservation of ultrastructural details. Indeed, for lower magnifications $(<10,000 x)$, it was still possible to carry out morphological analysis $(5000 \mathrm{x})$.

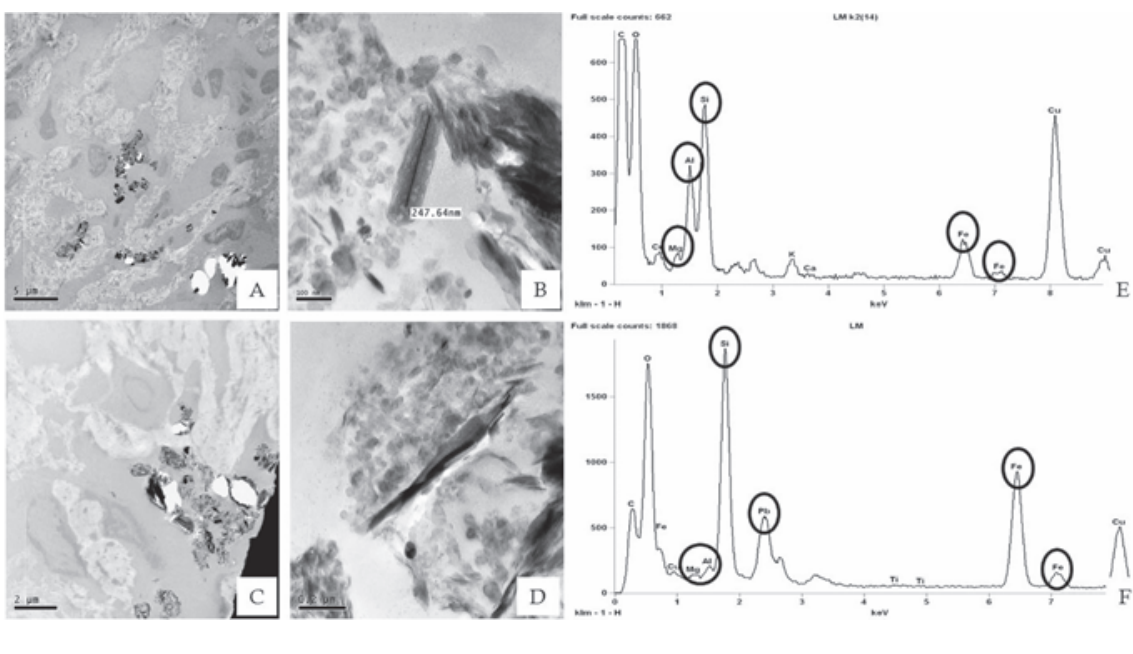

Figure 4. EDX microanalysis of asbestos fibers. TEM electron micrographs of 2 lung adeno-carcinoma processed by FSE method show numerous fibers with variable length, of 0.2-0.6 $\mu \mathrm{m}$ (A,B, case \#2; C,D, case \#4). EDX Microanalysis spectrum allowed us to identify these electrondense bodies as asbestos fibers, mainly composed of $\mathrm{Si}, \mathrm{Fe}, \mathrm{Mg}$ and Al (E-F). 
ly multi-factorial etiology of this cancer, whereas the causal relationship between asbestos exposure and mesothelioma is much more specific. $^{11}$

Lung cancer develops several decades after exposure to asbestos, so it may be difficult to obtain reliable documentation about the presence and extent of past exposure. The duration of assumed past exposure is often used as a surrogate; however, its predictive power for the future development of lung cancer has been recently questioned..$^{12}$ To circumvent these limitations, the histological detection of asbestos in lung cancer is used to support the attribution of lung cancer to asbestos exposure. The current criteria used for this purpose ${ }^{13}$ do not include the search for asbestos nanofibers and the spatial relationship of asbestos fibers with cancer cells. From a pathophysiological perspective, however, both these criteria should be relevant in the process of attribution. In fact, in recent years it has become clear that a material in the nanometric range may be more dangerous than the same material in bulk form. ${ }^{14-16}$ If this rule is universal, it should apply also to asbestos nanofibers. ${ }^{17}$ Additionaly, the process of malignant transformation of normal lung cells into malignant ones seems to require the direct contact of asbestos fibers with these cells, ${ }^{9}$ and therefore it would be logical that the presence or absence of asbestos fiber in the context of the neoplastic lesion should be considered in the process of the etiological attribution of the lesion itself. ${ }^{18}$

In this work, we evaluated the ability of EDX in unmasking asbestos fibers in the nanometric range, missed by conventional diagnostic techniques. For this purpose, we tested the FSE method. This method allowed us to unmask hidden asbestos fibers thanks to the correlation between H\&E-stained areas and those for TEM and EDX microanalysis and to the partially preserved cellular morphology. Asbestos nanofibers were not observed in the histological samples of lung cancer patients with no exposure to asbestos, and therefore the association seems to be highly specific, although, of course further data are needed. Noteworthy, the same specificity does not apply to current criteria for the evidence of asbestos exposure in lung cancer patients: fibers are searched in the whole lung, and a threshold is required, given their presence also in non-exposed patients. ${ }^{19}$

A potential limitation of FSE method is represented by the loss of ultrastructural details in paraffin-embedded tissues, which does not allow a magnification higher than $10,000 x$. However, we were able to perform a satisfactory morphological analysis and to identify fibers deposits. Indeed, as stated above, it is important to verify the presence of fibers in pathological tissue areas. Moreover, EDX micro- analysis identifies the composition of individual particles, which is often, in itself, sufficient to identify specific fiber isotype. In our work, thanks to EDX microanalysis, we detected a rare asbestiform variety of amosite, the antophyllite-gedrite. Fibrous anthophyllitegedrite is one of the six recognised types of asbestos widely used in asbestos cement and for insulation. The identification of the specific isotype of asbestos made possible to unequivocally associate the fibers detected into lung with the real occupational exposure of the patient. The asbestos fibers evidenced with our technique had not only a diameter in the nanometric range, but were also very short, with a length lower than $0.6 \mu \mathrm{m}$. It is of interest that a recent epidemiologic study detected a strong association between lung cancer and asbestos fibers shorter than 1.5 $\mu \mathrm{m} .{ }^{20}$ Another important feature of the method we used in this work is that it can be used to re-evaluate possible asbestos exposure in retrospective studies. Therefore, if the data of this study are confirmed by other laboratories, ultrastructural studies and EDX microanalysis on FFPE could offer exposed workers the chance to ask for re-evaluation of their case. Noteworthy, our protocol allows to identify asbestos fibers without lung digestion. This procedure results extremely useful since generally, in the clinical practice, histological diagnosis of lung cancer is performed on very small amount of tissue impeding the possibility to reserve part of it for the detection of asbestos fibers by digestion. Furthermore, digestion techniques require a lot of biological sample competing for specimen use for biological markers assessment or asbestos fibers detection. In conclusion, we proposed a new analytical protocol that can be a helpful trials to detect asbestos nanofibers also in retrospective analysis, using archival lung FFPE tissues. This method allows to unmask exposure to asbestos nano-fibers undetectable with conventional methods. The possibility of analyze FFPE tissue with TEM and EDX microanalysis can be contributed to reduce the wide underestimation of the casual link between asbestos and lung cancer. Furthermore, the revaluation of these biopsies can be a valid support instrument for medico-legal cases aimed at the resolution of work services.

\section{References}

1. Pietroiusti A. Health implications of engineered nanoparticles. Nanoscale 2012;4: 1231-47.

2. Shvedova A, Pietroiusti A, Fadeel B, Kagan V. Mechanisms of carbon nanotubeinduced toxicity: focus on oxidative stress.
Toxicol Appl 2012;261:121-33.

3. Sakamoto Y, Nakae D, Fukumori N, Tayama K, Maekawa A, Imai K, et al. Induction of mesothelioma by a single intrascrotal administration of multi wall carbon nanotubes in intact male Fischer 344 rats. J Toxicol Sci 2009;34:65-76.

4. Kaluza S, Balderhaar, Orythen B, Honnert B, Jankowska E, Pietrowski P, et al. Workplace exposures to nanoparticles. European Agency for Safety and Health at Work, Brussels, Belgium. EU-OSHA 2009.

5. Stayner L, Kuempel E, Gilbert S, Hein M, Dement J. An epidemiological study of the role of chrysotile asbestos fibre dimensions in determining respiratory disease risk in exposed workers. Occup Environ Med 2008;65:613-9.

6. Fox CH, Johnson FB, Whiting J, Roller PP. Formaldehyde fixation. J Histochem Cytochem 1985;33:845-53.

7. Scimeca M, Giannini E, Antonacci C, Pistolese CA, Spagnoli LG, Bonanno E. Microcalcifications in breast cancer: an active phenomenon mediated by epithelial cells with mesenchymal characteristics. BMC Cancer 2014;14:286.

8. Scimeca M, Orlandi A, Terrenato I, Bischetti S, Bonanno E. Assessment of metal contaminants in non-small cell lung cancer by EDX microanalysis. Eur J Histochem 2014;58:2403.

9. World Health Organization Cancer. Fact sheet no. 297 left fence. Accessed on: February 2015. Available from: http://www.who.int/mediacentre/factsheets/fs297/en/right

10. Świątkowska B, Szubert Z, Sobala W, Szeszenia-Dąbrowska N. Predictors of lung cancer among former asbestosexposed workers. Lung Cancer 2015;89: 243-8.

11. Bayram M, Bakan ND. Environmental exposure to asbestos: from geology to mesothelioma. Curr Opin Pulm Med 2014; 20:301-7.

12. Villeneuve PJ, Parent MÉ, Harris SA, Johnson KC. Occupational exposure to asbestos and lung cancer in men: evidence from a population-based case-control study in eight Canadian provinces. BMC Cancer 2012;12:595.

13. Wolff H, Vehmas T, Oksa P, Rantanen J, Vainio H. Asbestos, asbestosis, and cancer the Helsinki criteria for diagnosis and attribution 2014: recommendations. Scand J Work Environ Health 2015;41:5-15.

14. Horie M, Kato H, Endoh S, Fujita K, Komaba LK, Nishio K, et al. Cellular effects of industrial metal nanoparticles and hydrophilic carbon black dispersion. J Toxicol Sci 2014;39:897-907.

15. Lai X, Wei Y, Zhao H, Chen S, Bu X, Lu F, et 
al. The effect of Fe203 and Zn0 nanoparticles on cytotoxicity and glucose metabolism in lung epithelial cells. J Appl Toxicol 2015;35:651-64.

16. Sadeghi L, Yousefi Babadi V, Espanani HR. Toxic effects of the Fe203 nanoparticles on the liver and lung tissue. Bratisl Lek Listy 2015;116:373-8.

17. Dement JM, Kuempel ED, Zumwalde RD, Ristich AM, Fernback JE, Smith RJ. Airborne fiber size characterization in exposure estimation: Evaluation of a modified transmission electron microcopy protocol for asbestos and potential use for carbon nanotubes and nanofibers. Am J Ind Med 2015;58:494-508.

18. Ngamwong Y, Tangamornsuksan W, Lohitnavy 0, Chaiyakunapruk N, Scholfield CN, Reisfeld B, et al. Additive Synergism between Asbestos and Smoking in Lung Cancer Risk: A Systematic Review and Meta-Analysis. PLoS One 2015;10: e0135798.

19. Brody AR. Pulmonary cell interaction with asbestos fibers in vivo and in vitro. Chest 1986;89;155S-9.

20. Loomis D, Dement J, Richardson D, Wolf S. 2010. Asbestos fibre dimensions and lung cancer mortality among workers exposed to chrysotile. Occup Environ Med 2010;67:580-4. 